\title{
Hydrogen sulfide alleviates myocardial fibrosis in mice with alcoholic cardiomyopathy by downregulating autophagy
}

\author{
BIAO LIANG ${ }^{1 *}$, TING XIAO ${ }^{2 *}$, JUNRONG LONG $^{1}$, MAOJUN LIU ${ }^{1}$, \\ ZINING LI ${ }^{1}$, SHENGQUAN LIU ${ }^{1}$ and JUN YANG ${ }^{1}$ \\ ${ }^{1}$ Department of Cardiology, The First Affiliated Hospital of University of South China, Hengyang, Hunan 421001;
${ }^{2}$ Department of Cardiology, Shenzhen Longhua New District Central Hospital, Shenzhen, Guangdong 518110, P.R. China
}

Received March 3, 2017; Accepted September 26, 2017

DOI: $10.3892 /$ ijmm.2017.3191

\begin{abstract}
Myocardial fibrosis is one of the most important pathological features of alcoholic cardiomyopathy (ACM). Hydrogen sulfide $\left(\mathrm{H}_{2} \mathrm{~S}\right)$ exerts protective effects in various types of cardiovascular disease, which has been demonstrated by many previous studies. However, there is a lack of adequate research on the effect of $\mathrm{H}_{2} \mathrm{~S}$ on myocardial fibrosis in ACM. The present study aimed to investigate the etiopathogenic role of $\mathrm{H}_{2} \mathrm{~S}$ in myocardial fibrosis induced by chronic alcohol intake. An ACM mouse model was induced by consumption of $4 \%$ ethanol solution in drinking water for 12 weeks. Sodium hydrosulfide (NaHS) was used as a donor to provide exogenous $\mathrm{H}_{2} \mathrm{~S}$. Twelve weeks later, mice were sacrificed to calculate the heart to body weight ratio. The degree of myocardial collagen deposition was evaluated by Masson's and Van Gieson's staining, the expression level of collagen I was measured by immunohistochemistry and autophagosomes were observed by transmission electron microscopy. In addition, the expression levels of autophagy-associated proteins and fibrosis-associated proteins were detected by western blotting, and the expression levels of miR-21 and miR-211 were detected by reverse transcription-quantitative polymerase chain reaction. The outcomes of the study revealed that chronic alcohol intake results in myocardial fibrosis, enhanced myocardial collagen deposition and increased expression levels of collagen I, autophagy, autophagy-associated proteins (Beclin 1, Atg3 and Atg7) and fibrosis-associated proteins (MMP8, MMP13, MMP14, MMP17 and TGF- $\beta 1$ ), as well as miR-21 and miR-221. These results were markedly reversed following treatment with $\mathrm{H}_{2} \mathrm{~S}$.
\end{abstract}

Correspondence to: Professor Jun Yang, Department of Cardiology, The First Affiliated Hospital of University of South China, 69 Chuangshan Road, Shigu, Hengyang, Hunan 421001, P.R. China E-mail: yangjunincn@163.com

${ }^{*}$ Contributed equally

Key words: hydrogen sulfide, alcoholic cardiomyopathy, myocardial fibrosis, autophagy, microRNA
The present study confirmed that $\mathrm{H}_{2} \mathrm{~S}$ relieves myocardial fibrosis in mice with ACM, and the underlying mechanism may involve the downregulation of autophagy and miR-21 and miR-211 expression levels.

\section{Introduction}

Long-term alcohol abuse may cause chronic injuries to the myocardium and be further associated with an increased incidence of alcoholic cardiomyopathy (ACM). ACM refers to a specific cardiomyopathy that has frequently been observed in patients who have a history of long-term alcohol abuse, but excludes organic heart diseases, such as congenital heart disease, valvular heart disease, coronary heart disease, hypertension and myocarditis (1). Scholars hypothesize that almost all ACM patients exhibit varying degrees of myocardial remodeling, including the remodeling of cardiac collagen, i.e., myocardial fibrosis (2). Myocardial fibrosis, a major pathological process in myocardial remodeling, leads to impaired systolic and diastolic function, which is closely associated with the occurrence and development of chronic heart failure, arrhythmia or even sudden cardiac death. However, there remains no specific treatment that effectively alleviates myocardial fibrosis caused by long-term alcohol abuse. Therefore, in-depth investigations into the underlying mechanism of myocardial fibrosis in ACM are conducive to establish novel preventive and therapeutic strategies to alleviate myocardial fibrosis, and what is more, to reduce the risk of heart failure in ACM patients.

Autophagy is an evolutionarily conserved process and a key mechanism for the maintenance of cellular homeostasis, which has been associated with the degradation and recycling of damaged or unnecessary proteins and organelles to promote cell survival under stressful conditions. Autophagy has been demonstrated to be involved in a series of physiological and pathological processes $(3,4)$. Accumulating evidence indicates that fundamental autophagy is an essential cellular protective and cellular self-aid behavior in harsh environments, beyond this range, it will lead to cell damage or cell death. Excessive autophagy is stimulated via lysosome-driven degradations in response to a variety of extracellular and intracellular stresses, including deprivation of nutrients and growth factors. Numerous studies have confirmed that myocardial cells, stimulated by multiple pathological factors, such as ischemia 
reperfusion, high blood glucose and overload, are generally associated with excessively activated autophagy (5-7). High levels of autophagy causes a large loss of myocardial cells $(8,9)$, which is considered as a crucial event in the progression of myocardial remodeling and heart failure (10). Long-term alcohol intake may give rise to increasing expression levels of microtubule-associated protein 1 light chain 3 type II (LC3-II) and autophagy related 7 (Atg7), which are autophagy-associated proteins in heart tissues. By contrast, myocardial injuries caused by alcohol intake may be markedly improved when autophagy is inhibited by 3-methyladenine (3-MA) (11). The above-mentioned studies indicate a potential correlation between autophagy and alcohol-induced myocardial injuries. MicroRNA (miRNA), as a small non-coding endogenous RNA, causes degradation of messenger RNA (mRNA) or blocks the translation process of mRNA into protein, and participates in and regulates multiple biological processes, such as cell proliferation, differentiation and apoptosis. A previous study revealed that the variation in expression profiles of miRNA may be involved in the regulation of myocardial remodeling and cell autophagy (12), and extensive interactions have been observed between phosphatidylinositol-4,5-bisphosphate 3-kinase (PI3K)/AKT and transforming growth factor (TGF)- $\beta 1$, which are involved in the regulation of myocardial fibrosis, autophagy, miR-21 and miR-221. In a study on patients with ACM conducted by Jing et al (13), it was found that differential expression of miRNAs may also participate in the occurrence and development of ACM (13).

Hydrogen sulfide $\left(\mathrm{H}_{2} \mathrm{~S}\right)$ is recognized as the third most common endogenously produced gaseous signaling molecule in mammalian systems [the top two being nitric oxide (NO) and carbon monoxide (CO)]. Numerous studies have demonstrated its protective effect on the brain, liver, kidney, heart, lung and other organs. $\mathrm{H}_{2} \mathrm{~S}$ is well-known as a potent vasodilator and signaling molecule under normal and pathophysiological conditions (14). However, the protective effect of $\mathrm{H}_{2} \mathrm{~S}$ on ACM, as well as its intrinsic mechanism remain unknown. Studies have demonstrated that $\mathrm{H}_{2} \mathrm{~S}$ improves the left ventricular function of smoking rats by downregulating autophagy (15) and provides a protective effect on the brain in traumatic brain injuries (16). In a study of mouse models with myocardial ischemia or inflammatory injuries, it was identified that $\mathrm{H}_{2} \mathrm{~S}$ reduces ischemic injury and inhibits inflammatory responses in the myocardium of mice by upregulating the expression level of miRNA-21 (17). Thus, $\mathrm{H}_{2} \mathrm{~S}$ may alleviate myocardial fibrosis in ACM by regulating the expression levels of miRNA and cell autophagy; therefore, the present study established a mouse model of ACM to observe the effect of $\mathrm{H}_{2} \mathrm{~S}$ on myocardial fibrosis and cell autophagy in ACM. Furthermore, the mechanisms by which $\mathrm{H}_{2} \mathrm{~S}$ affected proteins in the PI3K/AKT signaling pathways involved in the regulation of autophagy and the expression levels of relevant miRNA were investigated.

\section{Materials and methods}

Animals and reagents. The experimental protocol was approved by the Animal Ethics Committee of the University of South China (Hengyang, China). Male Kunming mice ( $n=44$; weight, 16-21 g) were purchased from the Animal Experimental Center of the University of South China. Animals were housed separately, under a controlled temperature $\left(24^{\circ} \mathrm{C}\right)$ and a normal phase light-dark cycle (light from 8:00 a.m. to 8:00 p.m.), with free access to food and water or ethanol solution. Sodium hydrosulfide (NaHS) and D,L-propargylglycine (PAG) were purchased from Sigma-Aldrich; Merck KGaA (Darmstadt, Germany). Cell lysis buffer for western blotting, phenylmethanesulfonyl fluoride, a bicinchoninic acid (BCA) protein assay kit (cat. no. P0012), SDS-PAGE gel preparation kit (cat. no. P0012A) and chloral hydrate were all purchased from Beyotime Institute of Biotechnology (Shanghai, China). Polyvinylidene fluoride (PVDF) membranes and prestained color protein molecular weight marker were purchased from Sigma-Aldrich; MerckKGaA.Rabbitpolyclonalanti-glyceraldehyde3-phosphate dehydrogenase (GAPDH; cat. no. BM16243), rabbit polyclonal anti-matrix metalloproteinase 8 (MMP8; cat. no. BA2201), rabbit polyclonal anti-MMP13 (cat. no. BA2204), rabbit polyclonal anti-MMP14 (cat. no. BA1278), rabbit polyclonal anti-collagen I (cat. no. BA0325) and rabbit polyclonal anti-TGF- $\beta 1$ (cat. no. BA0290) were all purchased from Wuhan Boster Biological Technology, Ltd. (Wuhan, China). Rabbit polyclonal anti-MMP17 (cat. no. bs-1862R) and rabbit polyclonal anti-tissue inhibitor of metalloproteinase 1 (TIMP1; cat. no. bs-0415R) were both purchased from Beijing Biosynthesis Biotechnology Co., Ltd. (Beijing, China). In addition, rabbit monoclonal anti-Beclin 1 (cat. no. 3495), rabbit monoclonal anti-autophagy-related protein 3 (Atg3; cat. no. 3415), rabbit monoclonal anti-Atg7 (cat. no. 8558) and rabbit polyclonal anti-PI3K (cat. no. 4249) and rabbit polyclonal anti-AKT1 (cat. no. 75692) were all purchased from Cell Signaling Technology, Inc., (Danvers, MA, USA). Horseradish peroxidase-labeled goat anti-rabbit IgG (cat. no. 074-1506) was purchased from KPL, Inc. (Gaithersburg, MD, USA).

ACM model. Kunming male mice $(n=44)$ were divided randomly into the following four groups (n=11/group) subsequent to 7-day environment adaptive feeding: Control group, ACM model group (model group), ACM model with NaHS treatment group (NaHS group) and ACM model with PAG treatment group (PAG group). The ACM model was established via the drinking of $4 \%$ ethanol solution (used as the sole source of drinking water) freely for 12 weeks, according to the study by Ge and Ren (18) and mice in the control group had free access to clean water. The $4 \%$ ethanol solution and clean water were refreshed every morning. Mice in the NaHS group received a daily intraperitoneal injection of $50 \mu \mathrm{mol} / \mathrm{kg}$ NaHS, mice in the PAG group received a daily intraperitoneal injection of $40 \mathrm{mg} / \mathrm{kg}$ PAG (endogenous $\mathrm{H}_{2} \mathrm{~S}$ production enzyme inhibitor), and mice in the control and model groups were intraperitoneally injected with physiological saline of the same volume every day. Twelve weeks later, animals were sacrificed following anesthesia induced by chloral hydrate at a dose of $350 \mathrm{mg} / \mathrm{kg}$. Cardiac tissue samples were harvested for pathological sections, and morphological changes on the myocardium were observed via Masson's and Van Gieson's (VG) staining and used to judge whether the ACM model had been successfully established.

Histopathological examination. Myocardial tissue samples were fixed with $4 \%$ polyformaldehyde (Beyotime Institute of Biotechnology), washed with water, dehydrated with alcohol, 
embedded in paraffin (Beyotime Institute of Biotechnology) and sliced into sections (thickness, $4 \mu \mathrm{m}$ ). The sections were stained using a Masson's staining kit (cat. no. SBJ-0288) and VG staining kit (cat. no. SBJ-0297) (both from Nanjing Senbeijia Biological Technology Co., Ltd., Nanjing, China) according to the manufacturer's instructions and observed under a light microscope (Motic BA210; Motic Medical Diagnostic Systems Co., Ltd., Xiamen, China).

Transmission electronic microscopy observation. Left ventricular myocardial tissue samples were cut into slices and fixed with $2.5 \%$ glutaraldehyde (Sinopharm Chemical Reagent Co., Ltd., Shanghai, China), post-fixed with $1 \%$ osmium tetraoxide (Absin Bioscience Inc., Shanghai, China), rinsed with phosphoric acid rinse solution (Beyotime Institute of Biotechnology), dehydrated using a series of acetone (Beyotime Institute of Biotechnology) at different concentrations, embedded and solidified, and cut into slices (thickness, 50-100 nm). The ultrathin slices were stained using 3\% uranyl acetate (Shanghai Fortune Biological Technology Co., Ltd., Shanghai, China) and lead nitrate (Tanyun Industry Fine Chemical Co., Ltd., Yingkou, China) at $37^{\circ} \mathrm{C}$ for $30 \mathrm{~min}$. Samples were observed under a transmission electron microscopy and the images were saved.

Expression of collagen I detected by immunohistochemistry. Myocardial tissue samples were fixed with $4 \%$ polyformaldehyde, embedded in paraffin, cut into slices (thickness, $10 \mu \mathrm{m}$ ), dewaxed and hydrated. The slices were incubated with 3\% hydrogen peroxide (Beyotime Institute of Biotechnology), washed with phosphate-buffered saline (PBS; Beyotime Institute of Biotechnology), blocked with $10 \%$ normal goat serum (Beyotime Institute of Biotechnology) at $37^{\circ} \mathrm{C}$ for $10 \mathrm{~min}$, incubated at $37^{\circ} \mathrm{C}$ with rabbit polyclonal anti-collagen I (dilution 1:100) for $90 \mathrm{~min}$, washed with PBS, incubated at $37^{\circ} \mathrm{C}$ with secondary antibodies (dilution 1:2,000) for $15 \mathrm{~min}$, washed with PBS, incubated at $37^{\circ} \mathrm{C}$ with horseradish peroxidase-labeled streptavidin (Beyotime Institute of Biotechnology) for $20 \mathrm{~min}$, washed with PBS, colored with color developing reagent (Beyotime Institute of Biotechnology), stained with hematoxylin (Beyotime Institute of Biotechnology) at $37^{\circ} \mathrm{C}$ for $20 \mathrm{~min}$, dehydrated with alcohol and sealed with neutral resins.

Western blot analysis. Total proteins were extracted in cell dialysis buffer containing protease inhibitors and quantified using a BCA Protein Assay kit. The proteins were denatured, separated (40 $\mu \mathrm{g}$ protein/lane) by electrophoresis equipped with $10 \%$ SDS-PAGE and transferred to a PVDF membrane (200 mA, $110 \mathrm{~min}$ ). The membranes were blocked with Tris-buffered saline (Well-Biology Co., Ltd., Changsha, China) with Tween-20 (TBST; Beyotime Institute of Biotechnology) containing $5 \%$ skimmed milk at $37^{\circ} \mathrm{C}$ for $1 \mathrm{~h}$. The membranes were incubated overnight at $4^{\circ} \mathrm{C}$ with primary antibody diluted at the following appropriate concentrations: MMP8 (1:400), MMP13 (1:400), MMP14 (1:400), MMP17 (1:400), TIMP1 (1:400), TGF1 (1:400), Beclin 1 (1:1,000), Atg3 (1:1,000), Atg7 (1:1,000), PI3K (1:1,000) and AKT1 $(1: 1,000)$. Following washing with TBST buffer three times, the membranes were incubated with secondary antibody $(1: 8,000)$ for $1 \mathrm{~h}$ at $37^{\circ} \mathrm{C}$.
Table I. BW, HW and HW/BW ratio in each group (mean \pm SD).

\begin{tabular}{lrccc}
\hline Groups & Nos. & $\begin{array}{c}\text { BW } \\
(\mathrm{g})\end{array}$ & $\begin{array}{c}\text { HW } \\
(\mathrm{mg})\end{array}$ & $\begin{array}{c}\mathrm{HW} / \mathrm{BW} \\
(\mathrm{mg} / \mathrm{g})\end{array}$ \\
\hline Control & 11 & $43.96 \pm 3.58$ & $222.12 \pm 29.96$ & $5.06 \pm 0.60$ \\
Model & 9 & $42.37 \pm 4.18$ & $227.30 \pm 29.34$ & $5.36 \pm 0.43$ \\
$\begin{array}{l}\text { Sodium } \\
\text { hydrosulfide }\end{array}$ & 10 & $42.60 \pm 3.25$ & $221.20 \pm 36.94$ & $5.18 \pm 0.73$ \\
$\begin{array}{l}\text { L-propargylglycine } \\
\text { M }\end{array}$ & 9 & $40.97 \pm 3.10$ & $227.37 \pm 24.86$ & $5.57 \pm 0.71$ \\
\hline
\end{tabular}

BW, body weight; HW, heart weight.

Stripes were visualized using an enhanced chemiluminescence detection reagent (Wuhan Boster Biological Technology, Ltd., Wuhan, China) and analyzed using Alpha Imager 2200 (ProteinSimple, San Jose, CA, USA). GAPDH served as the internal reference.

Reverse transcription-quantitative polymerase chain reaction (RT-qPCR) analysis. Total RNA was extracted from myocardial tissue of mice in each group using TRIzol reagent (Invitrogen; Thermo Fisher Scientific, Inc., Waltham, MA, USA). Concentration of extracted RNA was measured using an ultraviolet spectrophotometer (Agilent Technologies, Inc., Santa Clara, CA, USA) and the integrity of RNA was analyzed with a gel imaging system (Bio-Rad Laboratories, Inc., Hercules, CA, USA). The RT reaction was manipulated with mi-RNA specific RT primer (GenScript Co., Ltd., Nanjing, China) using an RT polymerase chain reaction kit (cat. no. K1622; MBI Fermentas; Thermo Fisher Scientific, Inc.). RT-qPCR was performed and analyzed on a Thermo Scientific PikoReal RealTime PCR system (PikoReal 96; Thermo Fisher Scientific, Inc.). Expression levels of miR-21, miR-221, miR-133a and miR-199a were detected by qPCR using a Taqman mi-RNA assay probe (Applied Biosystems; Thermo Fisher Scientific, Inc.) and U6 was used as loading control for quantitation of miRNAs. RT was performed under the following conditions: $37^{\circ} \mathrm{C}$ for $15 \mathrm{~min}$, $42^{\circ} \mathrm{C}$ for $50 \mathrm{~min}$ and $85^{\circ} \mathrm{C}$ for $5 \mathrm{~min}$. The acquired cDNA was subjected to qPCR as follows: $50^{\circ} \mathrm{C}$ for $2 \mathrm{~min}, 95^{\circ} \mathrm{C}$ for $10 \mathrm{~min}$, $5 \mathrm{sec}$ at $95^{\circ} \mathrm{C}$ and $30 \mathrm{sec}$ at $60^{\circ} \mathrm{C}$ for 40 cycles. Relative quantification of miR-21, miR-221, miR-133a and miR-199a expression were calculated using the $2^{-\Delta \Delta \mathrm{Ct}}$ method.

Statistical analysis. Data are presented as the mean \pm standard deviation (mean $\pm \mathrm{SD}$ ). Differences among groups were evaluated by one-way analysis of variance using SPSS software (version 18.0; SPSS, Inc., Chicago, IL, USA). P<0.05 was considered to indicate a statistically significant difference.

\section{Results}

Effect of $\mathrm{H}_{2} \mathrm{~S}$ on body weight, heart weight and heart/body weight ratio. Twelve weeks after the intervention, the following numbers of mice had survived in each group: Control group, $\mathrm{n}=11$; model group, $\mathrm{n}=9$; NaHS group, $\mathrm{n}=10$; and PAG group, $\mathrm{n}=9$. As demonstrated in Table I, the differences of body weight, heart weight and heart/body weight ratio in each group were not statistically significant. 


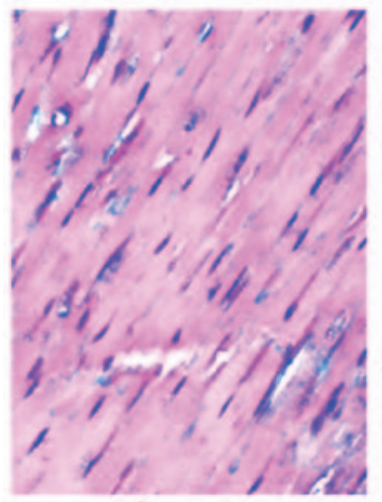

Control

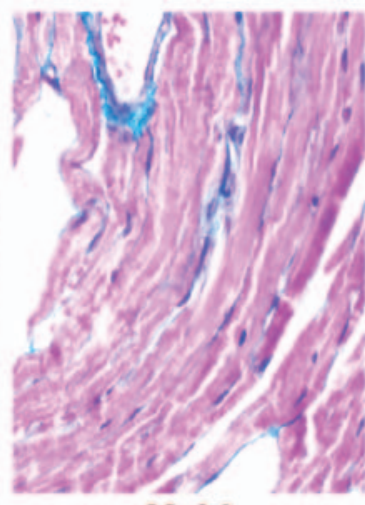

Model

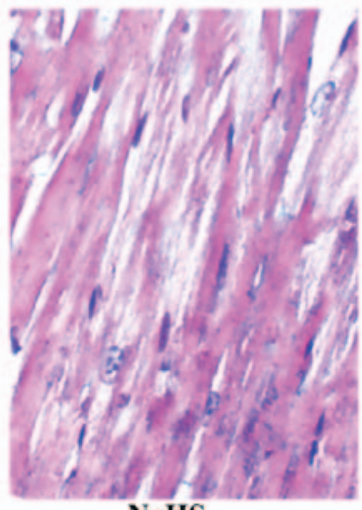

NaHS

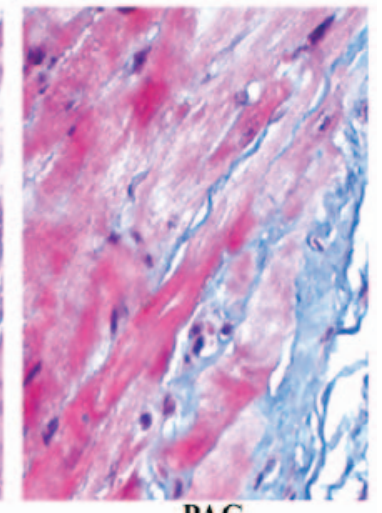

PAG

Figure 1. Masson's staining of myocardial tissue samples in each group. Irregularly arranged myocardial cells and increased fibrosis were observed in the model group compared with the control group. The degree of myocardial disarrangement and myocardial fibrosis were significantly reduced following treatment with $\mathrm{H}_{2} \mathrm{~S}$ in the NaHS group. Compared with the model group, aggravated myocardial disarrangement and myocardial fibrosis were observed in the PAG group. Magnification, x400. NaHS, sodium hydrosulfide; PAG, L-propargylglycine.

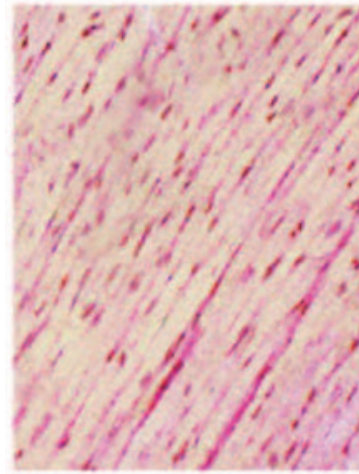

Control

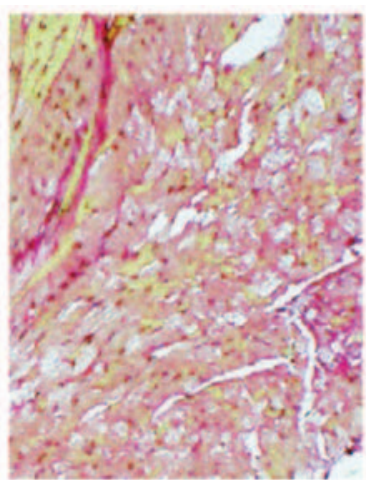

Model

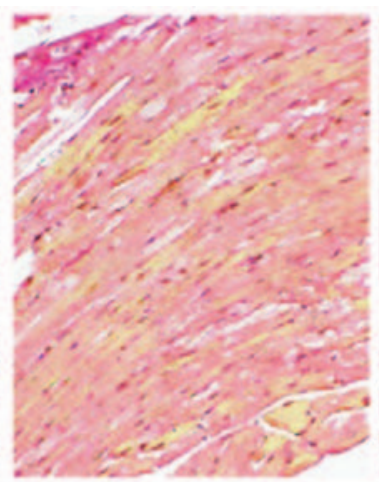

NaHS

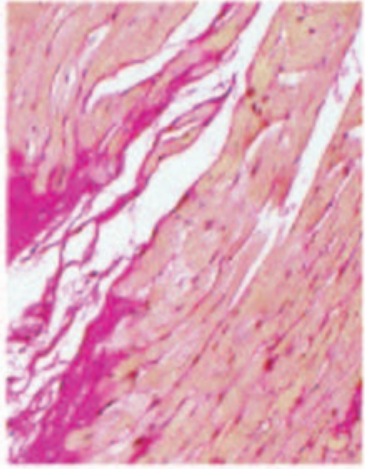

PAG

Figure 2. Van Gieson's staining of myocardial tissue samples in each group. Increased red-stained collagenous fibers were observed in the model group compared with the control group. In the NaHS group, myocardial collagen deposition was decreased compared with the model group. The degree of myocardial collagen deposition was exacerbated in the PAG group compared with the model group. Magnification, x100. NaHS, sodium hydrosulfide; PAG, L-propargylglycine.

Effect of $\mathrm{H}_{2} \mathrm{~S}$ on histopathological changes induced by ACM. In order to observe histopathological changes in myocardial tissue samples, Masson's staining was used and the blue staining indicated collagenous fibers in the myocardial tissue. As reflected in Fig. 1, irregularly arranged myocardial cells and increased fibrosis were observed in the model group compared with the control group. The degree of irregular arrangement of myocardial fibres and myocardial fibrosis were significantly reduced following treatment with $\mathrm{H}_{2} \mathrm{~S}$ in the NaHS group. In addition, compared with the model group, irregular arrangement of myocardial fibres and myocardial fibrosis were observed in the PAG group.

Effect of $\mathrm{H}_{2} \mathrm{~S}$ on myocardial collagen deposition. To evaluate the degree of myocardial collagen deposition, VG staining was performed and red staining indicated collagenous fibers in the myocardial tissue. As reflected in Fig. 2, increased red-stained collagenous fibers were observed in the model group compared with the control group. In the NaHS group, myocardial collagen deposition was decreased compared with in the model group. The degree of myocardial collagen deposition was exacerbated in the PAG group when compared with that in the model group.
Effect of $\mathrm{H}_{2} \mathrm{~S}$ on the expression level of collagen I. The expression level of collagen I was detected by immunohistochemistry to clarify myocardial fibrosis at the molecular level. As reflected in Fig. 3, the expression level of collagen I was markedly increased in the model group compared with that in the control group. Compared with the model group, the expression level of collagen I was markedly decreased in the NaHS group. Additionally, the expression level of collagen I was significantly increased in the PAG group compared with that in the model group.

Effect of $\mathrm{H}_{2} \mathrm{~S}$ on fibrosis-associated proteins. It is generally hypothesized that the degree of collagen synthesis and degradation is closely dependent on the balance of MMPs and TIMPs $(19,20)$. Furthermore, TGF- $\beta 1$ is regarded as a protein closely associated with myocardial fibrosis. For these reasons, the expression levels of MMP8, MMP13, MMP14, MMP17, TIMP1 and TGF- $\beta 1$ were detected by western blotting (Fig. 4). The results indicated that the expression levels of MMP8, MMP13, MMP14, MMP17 and TGF- $\beta 1$ were significantly increased, while the expression level of TIMP1 was significantly decreased in the model group, compared with the control group $(\mathrm{P}<0.05)$. These changes were significantly reversed in the NaHS group $(\mathrm{P}<0.05)$. Furthermore, compared 


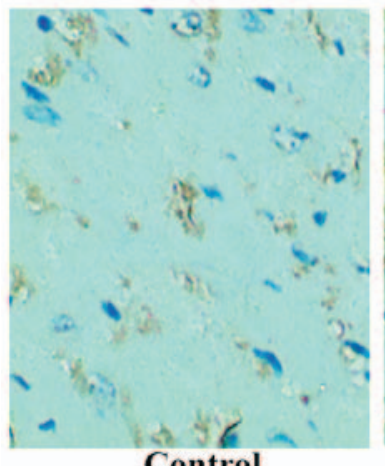

Control

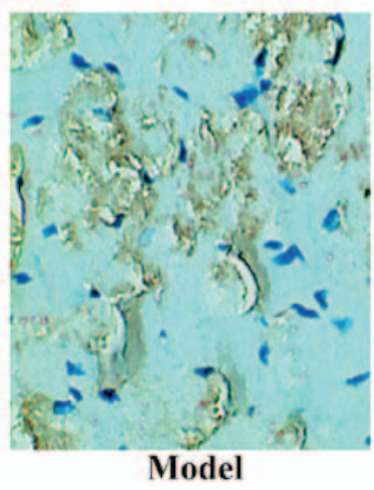

Model

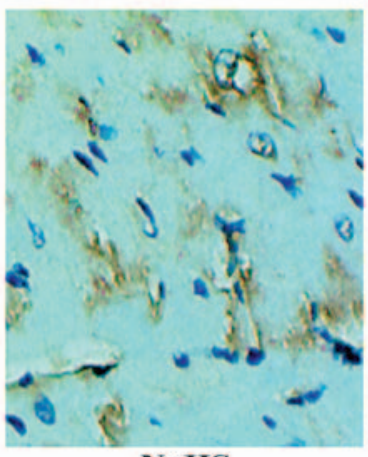

NaHS

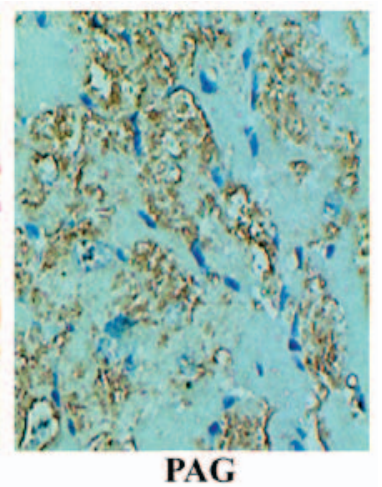

PAG

Figure 3. Immunohistochemical staining of collagen I in each group. The expression level of collagen I was markedly increased in the model group compared with the control group. Compared with the model group, the expression level of collagen I was markedly decreased in the NaHS group. The expression level of collagen I was significantly increased in the PAG group compared with the model group. Magnification, x400. NaHS, sodium hydrosulfide; PAG, L-propargylglycine.
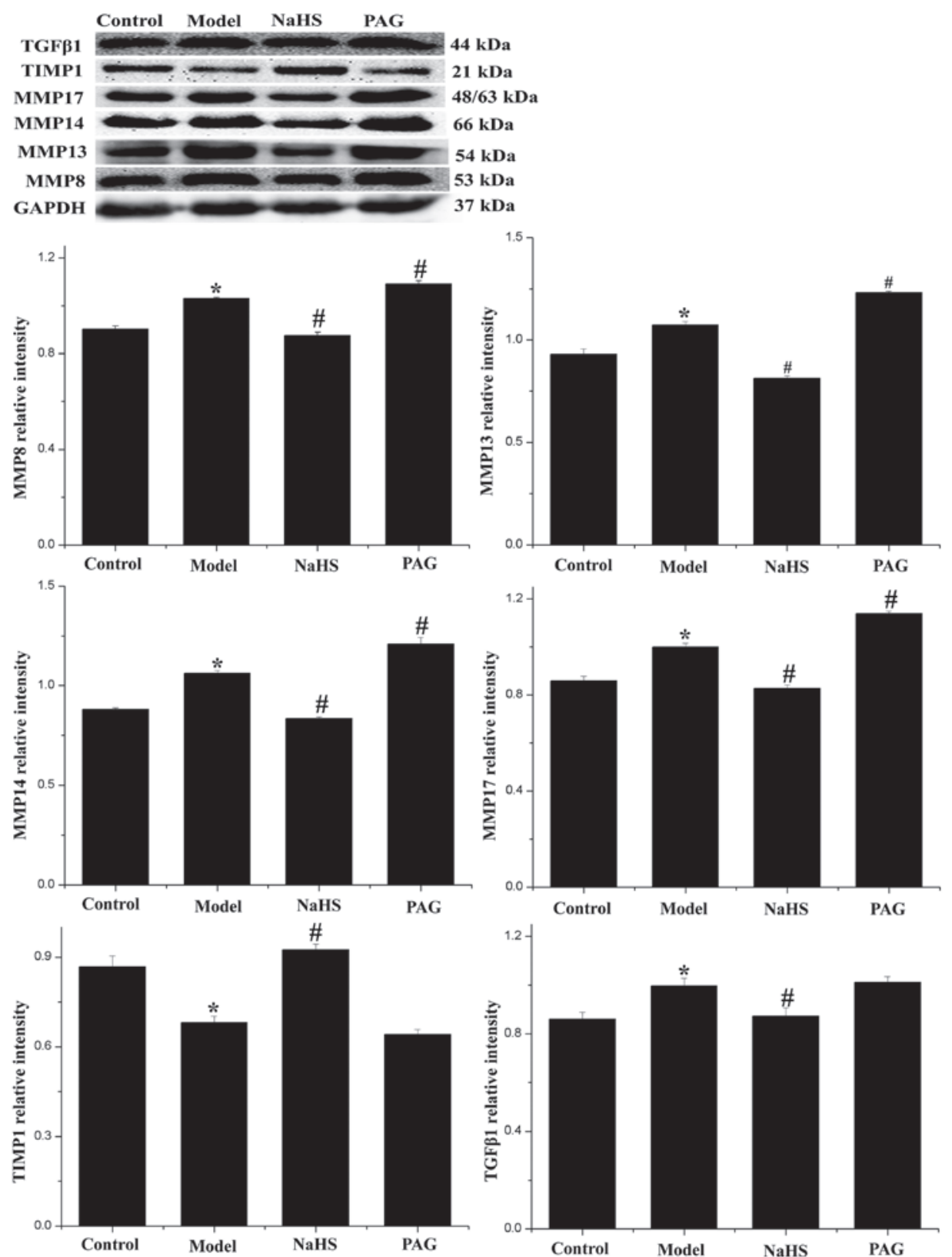

Figure 4. Expression levels of MMPs, TIMP1 and TGF- $\beta 1$ in myocardial tissue samples from each group. Means \pm standard deviation $(\mathrm{n}=3)$. ${ }^{*} \mathrm{P}<0.05 \mathrm{vs.}$ control group; ${ }^{~} \mathrm{P}<0.05$ vs. model group. MMP, matrix metalloproteinase; TIMP1, tissue inhibitor of metalloproteinase 1 ; TGF- $\beta 1$, transforming growth factor- $\beta 1$; GAPDH, glyceraldehyde 3-phosphate dehydrogenase; NaHS, sodium hydrosulfide; PAG, L-propargylglycine. 


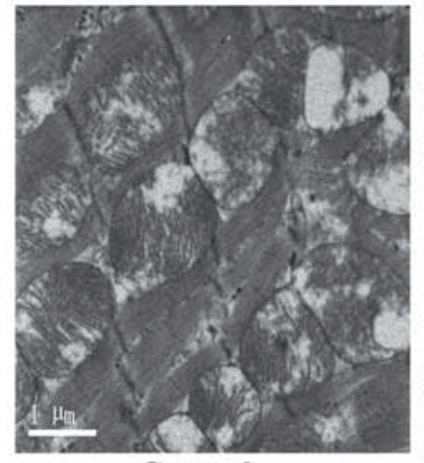

Control

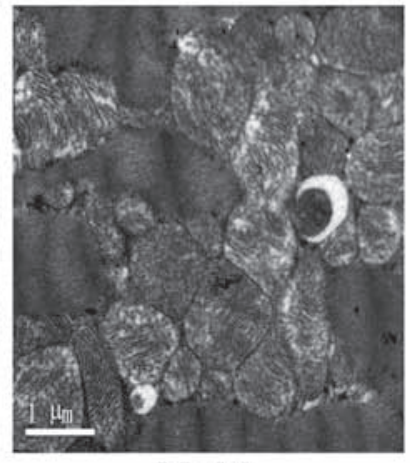

Model

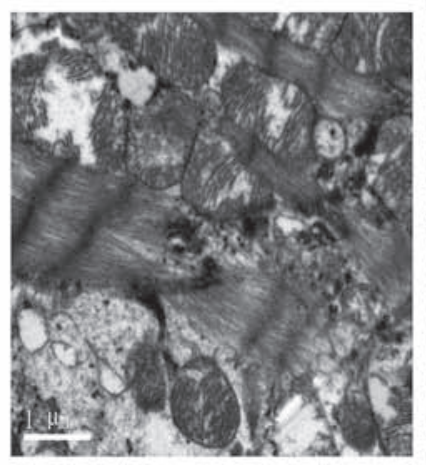

NaHS

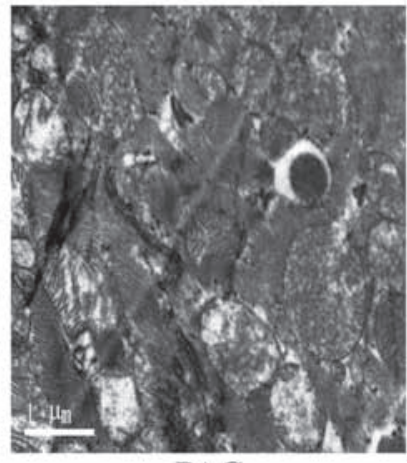

PAG

Figure 5. Observations of transmission electron microscope of myocardial tissue samples from each group. NaHS, sodium hydrosulfide; PAG, L-propargylglycine.
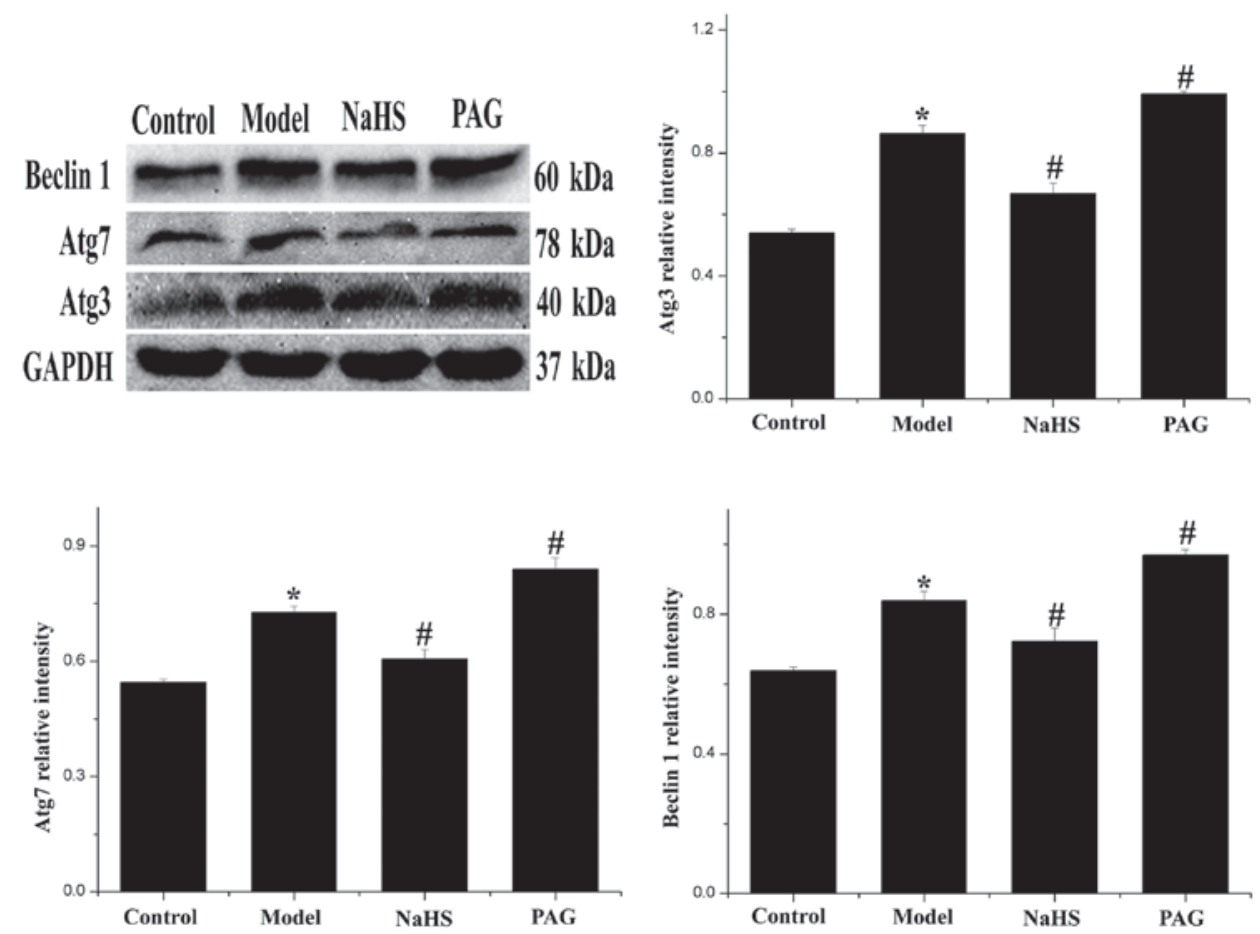

Figure 6. Expression levels of Beclin 1, Atg3 and Atg7 in myocardial tissue samples from each group. Means \pm standard deviation ( $\mathrm{n}=3$ ). ${ }^{*} \mathrm{P}<0.05$ vs. control group; ${ }^{\#} \mathrm{P}<0.05$ vs. model group. Atg, autophagy related; GAPDH, glyceraldehyde 3-phosphate dehydrogenase; NaHS, sodium hydrosulfide; PAG, L-propargylglycine.

with the model group, the expression levels of MMP8, MMP13, MMP14 and MMP17 were significantly increased $(\mathrm{P}<0.05)$, the expression level of TGF- $\beta 1$ was marginally increased, and the expression level of TIMP1 was slightly decreased $(\mathrm{P}>0.05)$ in the PAG group.

Effect of $\mathrm{H}_{2}$ S on cardiomyocyte autophagy. In order to evaluate autophagy level changes and the effect of $\mathrm{H}_{2} \mathrm{~S}$ on alcoholinduced autophagy in myocardial tissue samples of mice with long-term alcohol intake, transmission electron microscope was used to observe the autophagosomes in each group. As demonstrated in Fig. 5, autophagosomes were observed in the model and PAG groups, and no observable autophagosomes were identified in the control and NaHS groups.

Effect of $\mathrm{H}_{2} \mathrm{~S}$ on expression levels of autophagy-associated proteins. In order to further clarify the level of autophagy in myocardial tissue, autophagy-associated proteins, Beclin 1, Atg3 and Atg7 were detected by western blotting. As revealed in Fig. 6, expression levels of Beclin 1, Atg3 and Atg7 were significantly increased in the model group compared with the control group $(\mathrm{P}<0.05)$. After treatment with $\mathrm{H}_{2} \mathrm{~S}$, expression levels of Beclin 1, Atg3 and Atg7 were significantly decreased $(\mathrm{P}<0.05)$. Furthermore, compared with the model group, expression levels of Beclin 1, Atg3 and Atg7 were significantly increased in the PAG group $(\mathrm{P}<0.05)$.

Effect of $\mathrm{H}_{2} \mathrm{~S}$ on PI3K/AKT1 signaling expression. The expression levels of PI3K and AKT1 were detected by western blotting and demonstrated PI3K/AKT1 as a classical signaling pathway in the regulation of autophagy (Fig. 7). Compared with the control group, the expression levels of PI3K and AKT1 were significantly decreased in the model group $(\mathrm{P}<0.05)$. In addition, changes were significantly reversed following treat- 

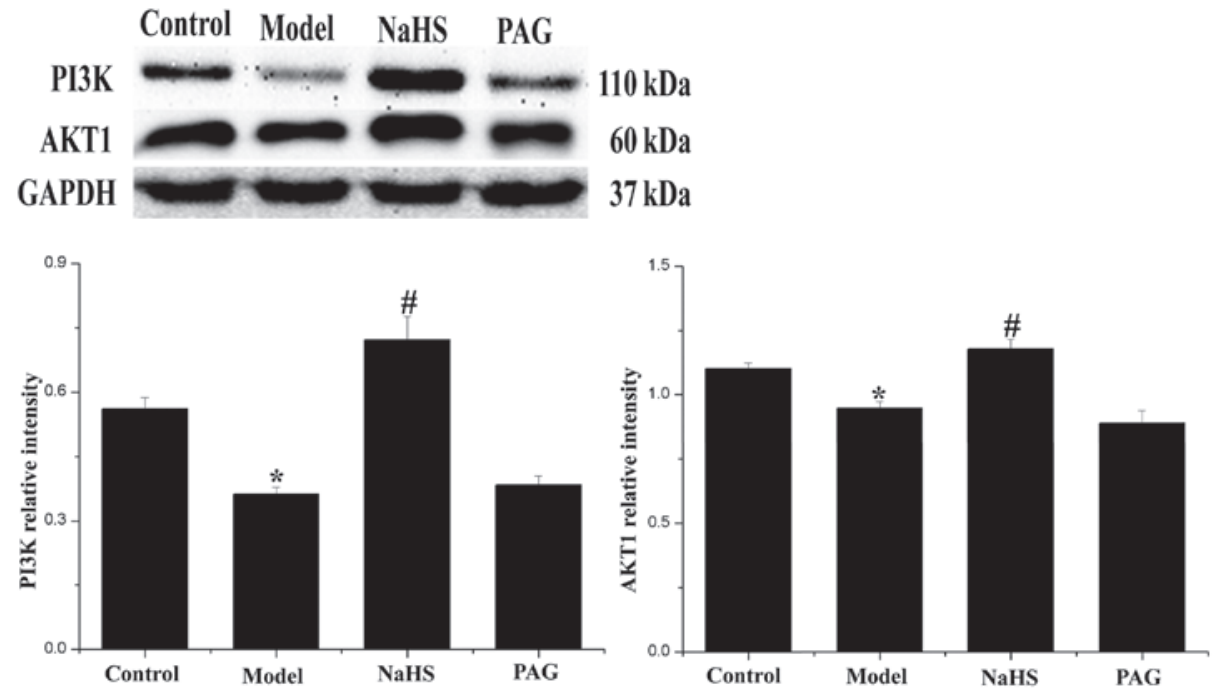

Figure 7. Expression levels of PI3K and AKT1 in myocardial tissue samples from each group. Means \pm standard deviation ( $\mathrm{n}=3$ ). ${ }^{\mathrm{P}}<0.05$ vs. control group; ${ }^{\#} \mathrm{P}<0.05$ vs. model group. PI3K, phosphatidylinositol-4,5-bisphosphate 3-kinase; GAPDH, glyceraldehyde 3-phosphate dehydrogenase; NaHS, sodium hydrosulfide; PAG, L-propargylglycine.
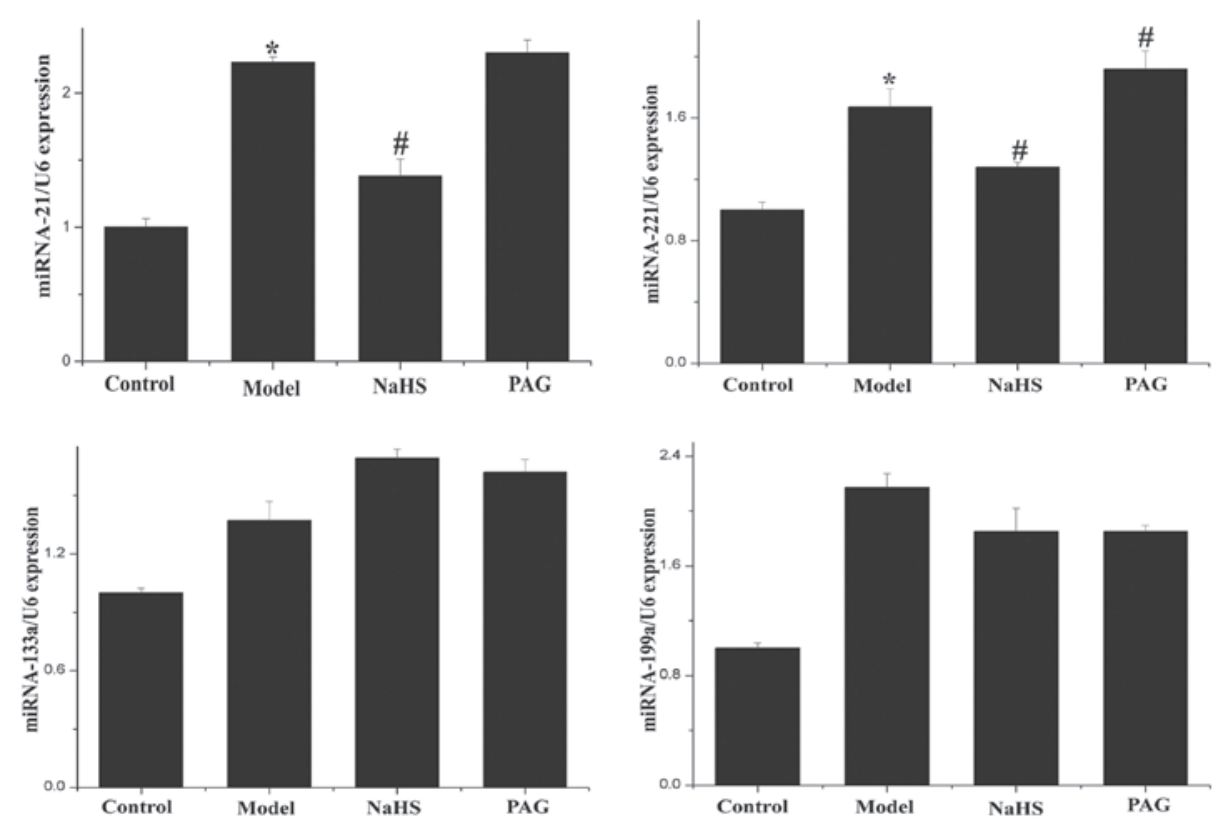

Figure 8. Expression levels of miR-21, miR-221, miR-133a and miR-199a in myocardial tissues from each group. Means \pm standard deviation ( $\mathrm{n}=3$ ). ${ }^{*} \mathrm{P}<0.05 \mathrm{vs}$. control group; " $\mathrm{P}<0.05$ vs. model group. miR, microRNA; NaHS, sodium hydrosulfide; PAG, L-propargylglycine.

ment with $\mathrm{H}_{2} \mathrm{~S}$ in the NaHS group $(\mathrm{P}<0.05)$. Compared with the model group, the expression levels of PI3K and AKT1 were not significantly decreased in the PAG group ( $\mathrm{P}>0.05)$.

Effect of $\mathrm{H}_{2}$ S on expression levels of miR-21, miR-221, miR-133a and miR-199a. RT-qPCR was used to detect the expression levels of miR-21, miR-221, miR-133a and miR-199a in myocardial tissue samples. As shown in Fig. 8, expression levels of miR-21 and miR-221 were significantly increased in the model group compared with the control group $(\mathrm{P}<0.05)$. Following treatment with $\mathrm{H}_{2} \mathrm{~S}$, the expression levels of miR-21 and miR-221 were significantly decreased $(\mathrm{P}<0.05)$. Furthermore, compared with the model group, the level of miR-221 expression was significantly increased in the PAG group $(\mathrm{P}<0.05)$. The level of miR-21 expression was marginally greater in the PAG group than that in the model group, although the difference was not statistically significant $(P>0.05)$. The differences between miR-133a and miR-199a expression levels in each group were not identified to be statistically significant.

\section{Discussion}

Currently, the incidence rate of ACM has been increasing gradually as a result of wide-spread alcohol abuse, which renders ACM a major cause for heart failure and sudden cardiac death in clinical practices $(21,22)$. During the occurrence and development of ACM, various pathological changes may occur in the myocardium, including myocardial fibrosis. Existing 
studies have indicated that myocardial fibrosis is a critical link in the pathogenesis of ACM. For example, Law et al observed the evident myocardial fibrosis in the twelfth and 14th week after mice were administered with $4 \%$ ethanol aqueous solution (23). In addition, previous studies reported that pathological variations, such as cardiac hypertrophy and myocardial fibrosis, are observed in the myocardium of certain ACM patients with no symptoms (24). Myocardial fibrosis refers to the accumulation of excessive collagen fibers in the myocardial interstitium, the disproportional ratio of all types of collagens and the disorganized arrangement of collagen fibers (25). Many types of cardiovascular disease, such as cardiomyopathy, hypertension and coronary heart disease, exhibit obvious myocardial fibrosis in the advanced stage of the disease course, which is one of the major pathological features of myocardial remodeling. Myocardial remodeling induced by myocardial fibrosis is closely associated with the pathogenesis of cardiac insufficiency and cardiac arrhythmia, and is, therefore, a key link determining the disease outcome and an important therapeutic target. In the present study, myocardial fibers were observed to be disorganized in mice with chronic alcohol intake in large quantities, and evident myocardial collagen accumulation was also identified in the myocardial tissues of mice in the model group according to the Masson's staining and VG staining. In addition, immunohistochemistry revealed that the expression level of collagen I in the model group was significantly increased compared with that in the control group. MMPs are an important factor regulating the collagen accumulation of extracellular matrix collagen, and TIMPs, as the specific inhibitors of MMPs, may regulate the activity of MMPs, and the two jointly contribute to regulating the synthesis and degradation of collagen in myocardial tissues (26). Existing studies have revealed that upregulated expression levels of MMPs and downregulated expression levels of TIMPs may result in the synthesis of collagen in the myocardial interstitium and increase accumulation, further leading to the occurrence of myocardial fibrosis (27-29). In the present study, at the molecular level, the results indicated that protein expression levels of MMP8, MMP13, MMP14 and MMP17 were significantly elevated in the myocardial tissue samples of mice in the model group, and the protein expression level of TIMP1 was markedly decreased, indicating that the disproportionate MMPs/TIMP1 contributed to the occurrence of myocardial fibrosis in mice with chronic alcohol intake in large quantities.

$\mathrm{H}_{2} \mathrm{~S}$, the third most common internal gas signaling molecule and, like $\mathrm{CO}$ and NO, is characterized as a type of inorganic gas molecule with liposolubility that freely penetrates the cell membrane. Endogenous $\mathrm{H}_{2} \mathrm{~S}$ is generated by L-cysteine under the catalytic effect of pyridoxal phosphate-5-phosphate-dependent enzyme, although endogenous $\mathrm{H}_{2} \mathrm{~S}$ is usually produced by L-cysteine catalyzed by cystathionine $\gamma$-lyase (CSE). It has been confirmed that $\mathrm{H}_{2} \mathrm{~S}$ participates in the regulation of various physiological and pathological processes of the cardiovascular system, and certain studies revealed that $\mathrm{H}_{2} \mathrm{~S}$ exerts an important protective role in many cardiac injury models, including viral myocarditis, myocardial ischemia reperfusion injury and diabetic cardiomyopathy (30-33). In addition, a previous study reported that $\mathrm{H}_{2} \mathrm{~S}$ alleviates the remodeling of the left ventricle in rats with a history of long-term alcohol intake (34). In the present study, taking NaHS as the donor of $\mathrm{H}_{2} \mathrm{~S}$, an intervention was performed using $\mathrm{H}_{2} \mathrm{~S}$ against myocardial fibrosis in mice with chronic alcohol intake in large quantities. The results demonstrated that, following intervention with exogenous $\mathrm{H}_{2} \mathrm{~S}$, a significant alleviation in the accumulation of collagen fibers was observed in the myocardium, and obvious decreases were identified in the expression level of collagen I, and the expression levels of MMP8, MMP13, MMP14 and MMP17 were significantly downregulated. In addition, the expression level of TIMP1 was upregulated, indicating a marked improvement in the disproportionate MMPs/TIMP1. By contrast, PAG, the irreversible inhibitor of CSE, was used to inhibit the generation of endogenous $\mathrm{H}_{2} \mathrm{~S}$ (35), and following the intervention with PAG in mice with chronic alcohol intake in large quantities in the PAG group, the accumulation of collagen fibers in the myocardium was identified to be severely exacerbated, the expression of type-I collagen was upregulated, and the disproportionate tendency between MMPs and TIMP1 became more evident. These results indicate that $\mathrm{H}_{2} \mathrm{~S}$, a gaseous signaling molecule, may be involved in the regulation of ACM.

Autophagy has been demonstrated to be involved in various physiological and pathological processes. Autophagy is a key mechanism for the maintenance of cellular homeostasis, by which damaged organelles and unused proteins are destroyed and recycled to relieve cellular stress and provide nutrients to promote cell survival (36). When excessive autophagy destroys the cytosol and organelles beyond a certain threshold, autophagic cell death is induced, together with apoptosis and necrosis (37). Matsui et al found that the process of autophagy is activated in response to energy crisis and oxidative stress under the condition of cardiac ischemia reperfusion injury. Although autophagy is protective during ischemia, it is detrimental during reperfusion (38). The roles of autophagy in the occurrence and development of ACM, i.e., protecting the cells from injuries or exacerbating the injuries, predominantly depend on the position of autophagy and the disease course, which is subsequently dependent on the profile and the strength of stimulations given to cells (39). Overwhelming autophagy may cause the death of autophagic cells. The loss of myocardial cells and the augmentation of components in the extracellular matrix could further facilitate pathological myocardial remodeling. A previous study demonstrated that the autophagy level was significantly elevated in the myocardium of transgenic mice that were overexpressing Beclin 1 and under pressure-overload, and may also cause pathological myocardial remodeling, although inhibiting autophagy may markedly alleviate myocardial remodeling (40). In addition, Miyata et al (41) identified that granulocyte colony-stimulating factor may decrease fibrosis in the myocardial interstitium by suppressing the autophagy level in myocardial cells. These studies indicated that the upregulation of autophagy is closely correlated with myocardial remodeling. A recent study reported the association between autophagy and ACM, and that the expression levels of LC3-II and Atg7, the autophagy-associated proteins, were significantly elevated in the heart tissues of murine with a history of long-term alcohol intake (11). Yet, $\mathrm{H}_{2} \mathrm{~S}$ was also reported by previous studies to participate in the regulation of cell autophagy (42). In addition, $\mathrm{H}_{2} \mathrm{~S}$ has been identified to remit myocardial ischemia reperfusion injury (43). While in the study performed by Zhou et al, $\mathrm{H}_{2} \mathrm{~S}$ 
improved the cardiac function of smoking rats by downregulating cell autophagy in the myocardium (15). Results in the present study indicated that the levels of autophagy-associated protein expression, such as Beclin 1, Atg3 and Atg7, of mice in the model group with long-term alcohol intake were significantly higher than those in the control group, and the autophagosomes were observed under the transmission electron microscope. Expression levels of Beclin 1, Atg3 and Atg7 were significantly decreased following treatment with NaHS, indicating that $\mathrm{H}_{2} \mathrm{~S}$ may downregulate the autophagy that was excessively activated in the myocardial tissues of mouse with a history of long-term alcohol intake. These results indicated that endogenous $\mathrm{H}_{2} \mathrm{~S}$ may participate in the regulation mechanisms of ACM and myocardial fibrosis resulting from long-term alcohol intake by regulating cell autophagy.

It has been found that the PI3K/AKT1 signaling pathway and TGF- $\beta$ signaling pathway are involved in the regulation of myocardial fibrosis and closely associated with the regulation mechanism of autophagy (44). Lin et al revealed that basic fibroblast growth factor (bFGF) improved heart function recovery and increased the survival of cardiomyocytes in a myocardial ischemia/reperfusion (I/R) model. The role of bFGF in myocardial $\mathrm{I} / \mathrm{R}$ recovery is associated with the inhibition of excessive autophagy via activation of the PI3K/Akt/mTOR signaling (45). Wang et al found that HDL ameliorated mechanical stress-induced cardiac hypertrophy and autophagy via Akt-dependent mechanism (46). These results indicate that the regulatory mechanism of autophagy is closely associated with PI3K/AKT signaling pathway. A vital checkpoint that negatively regulates autophagy is the mechanistic target of rapamycin (mTOR), which is the downstream target of the PI3K/AKT signaling pathway. Downregulation of the PI3K/AKT1 signaling pathway has been demonstrated to activate autophagy (47). In the present study, the PI3K/AKT1 signaling pathway was markedly inhibited in the myocardium of the model group, whereas $\mathrm{H}_{2} \mathrm{~S}$ treatment was observed to activate PI3K/AKT1 in mice following chronic alcohol exposure, which indicated that $\mathrm{H}_{2} \mathrm{~S}$ may protect against cardiac autophagy induced by chronic alcohol exposure via regulation of the PI3K/AKT1 signaling pathway. In the present study, the results indicated that a significant decrease was identified in the expression levels of PI3K/AKT in the myocardium of mice in the PAG group, which was similar to the model group, indicating that regulation of $\mathrm{H}_{2} \mathrm{~S}$ in autophagy, via the PI3K/AKT signaling pathway, may be the major mechanism by which the myocardium could be protected from the fibrosis induced by long-term alcohol intake. Similar with PI3K/AKT, TGF- $\beta 1$ is also critical in the pathogenesis of myocardial fibrosis and the two are common signaling pathways involved in the regulatory mechanism of autophagy. TGF- $\beta 1$ is central in the pathogenesis of myocardial fibrosis. TGF- $\beta 1$ promotes the occurrence of myocardial fibrosis by activating fibroblast hyperplasia and promoting the accumulation of collagen in the extracellular matrix (48). A previous study identified that the expression level of TGF- $\beta 1$ was significantly increased in the myocardium of a chronic iron overloaded mouse model with myocardial fibrosis (49), and a significantly increased expression level of TGF- $\beta 1$ was identified in the myocardial fibrosis induced by homocysteine (50). Upregulation of TGF- $\beta 1$ may result in widespread myocardial fibrosis with the induction of autophagy (51). The results in the present study indicated that the mechanism of myocardial interstitial fibrosis in mice following chronic alcohol exposure may be associated with the activation of TGF- $\beta 1$ signaling pathways as the autophagy level increases.

miRNAs are a class of naturally occurring, endogenous small non-coding RNA molecules that provide a mechanism for negative regulation of mRNA translation into proteins. miRNAs are distinct from, but associated with siRNAs and regulate their targets by either inhibiting mRNA translation or promoting mRNA degradation (52). Previous studies have indicated that miRNAs are essential in a number of biological processes, including proliferation, differentiation, apoptosis and development (53-55). Furthermore, the dysregulation of miRNAs has been linked to various pathological settings and their roles in cardiovascular diseases have been an area of intense investigation (56). A variety of studies have confirmed that miRNAs perform important regulatory roles in the occurrence and development of many cardiovascular diseases. Previous studies have identified that miRNAs are key regulators of genes involved in the pathophysiology of fibrosis in the heart (57). Increasing evidence has demonstrated that several miRNAs, particularly, miR-21, miR-221, miR-133a and miR-199a, have been implicated in the control of myocardial fibrosis and reported to be closely associated with the mechanism of myocardial remodeling (58). Thum et al found that the expression level of miR-21 was significantly elevated in a heart failure model; however, silenced miR-21 may alleviate myocardial fibrosis and improve heart function (59). In addition, further studies revealed that miR-21 initiates the extracellular signal-regulated kinases-mitogen-activated protein kinase signaling pathway by inhibiting the target gene, sprouty RTK signaling antagonist 1 . This promotes the proliferation of fibroblasts and relevant excretion of growth factors, thus accelerating the disease course of myocardial fibrosis and myocardial remodeling. Furthermore, the profibrogenic mechanism of miR-21 may be involved in regulating the expression of TGF- $\beta$ RIII, which is a negative regulation factor in the TGF- $\beta$ signaling pathway that inhibits the occurrence of myocardial fibrosis. By experimenting on myocardial infarction models, Liang et al confirmed that TGF- $\beta$ RIII is the target gene of miRNA-21 and the overexpression of miRNA-21 may inhibit the expression of TGF- $\beta$ RIII to initiate the TGF- $\beta$ signaling pathway to increase the content of collagen fibers (60). In the study by Wang et al, it was found that overexpression of miR-221 induces the hypertrophy of myocardial cells (61). However, certain studies have indicated that miRNA-221 may specifically inhibit the expression of P27 and phosphatase and tensin homolog genes (62), participate in the regulation of the PI3K/AKT signaling pathway and cell autophagy (63), and intimated that crosstalk exists between miRNA-21/miRNA-221 and TGF- $\beta$, as well as PI3K/AKT signaling pathways, which jointly participate in the regulation mechanism of cell autophagy and myocardial fibrosis $(64,65)$. The results of the present study demonstrated that the expression levels of miR-21 and miR-221 in the model group were significantly higher than those in the control group; while compared with the model group, the expression levels in the NaHS group were significantly downregulated. By contrast, the expression level of miR-221 in the PAG group was signifi- 
cantly higher than that in the model group, indicating that the pathogenesis of ACM may be correlated with the upregulated expression levels of miR-21 and miR-221. The abnormal expression in miR-21 and miR-221 that regulates the miRNA may be the other mechanism by which $\mathrm{H}_{2} \mathrm{~S}$ improves myocardial fibrosis following long-term alcohol consumption.

In conclusion, the results of the present study indicated that $\mathrm{H}_{2} \mathrm{~S}$ inhibits cell autophagy and alleviates myocardial fibrosis by regulating the expression levels of miR-21 and miR-221 via the TGF- $\beta$ and PI3K/AKT signaling pathways, which contributes to the research regarding the pathogenesis of myocardial fibrosis in ACM and provides a novel idea for the clinical treatment of ACM. However, the specific mechanism of crosstalk between miRNA and TGF- $\beta$ and PI3K/AKT signaling pathways remains unknown in the occurrence and development of ACM, and further studies are required to investigate the clinical value and significance of the regulatory effect of $\mathrm{H}_{2} \mathrm{~S}$ on cell autophagy for the improvement of ACM and myocardial fibrosis.

\section{Acknowledgements}

The present study was supported by National Natural Science Foundation of China (grant no. 81270181).

\section{References}

1. Piano MR and Phillips SA: Alcoholic cardiomyopathy: Pathophysiologic insights. Cardiovasc Toxicol 14: 291-308, 2014.

2. Piano MR: Alcoholic cardiomyopathy: Incidence, clinical characteristics, and pathophysiology. Chest 121: 1638-1650, 2002.

3. Basu S, Rajakaruna S, Reyes B, Van Bockstaele E and Menko AS: Suppression of MAPK/JNK-MTORC1 signaling leads to premature loss of organelles and nuclei by autophagy during terminal differentiation of lens fiber cells. Autophagy 10: 1193-1211, 2014

4. Gottlieb RA and Mentzer RM Jr: Autophagy: An affair of the heart. Heart Fail Rev 18: 575-584, 2013.

5. Jian J, Xuan F, Qin F and Huang R: Bauhinia championii flavone inhibits apoptosis and autophagy via the PI3K/Akt pathway in myocardial ischemia/reperfusion injury in rats. Drug Des Devel Ther 9: 5933-5945, 2015.

6. Younce CW, Wang K and Kolattukudy PE: Hyperglycaemia-induced cardiomyocyte death is mediated via MCP-1 production and induction of a novel zinc-finger protein MCPIP. Cardiovasc Res 87: 665-674, 2010.

7. Zhu H, Rothermel BA and Hill JA: Autophagy in load-induced heart disease. Methods Enzymol 453: 343-363, 2009.

8. Ma S, Wang Y, Chen Y and Cao F: The role of the autophagy in myocardial ischemia/reperfusion injury. Biochim Biophys Acta 1852: 271-276, 2015.

9. Jie X, Qin X, Cai X, Yang L, Xing Y, Li J, Zhang L, Tang Y, Liu J, Zhang X, et al: Mitochondrial JNK activation triggers autophagy and apoptosis and aggravates myocardial injury following ischemia/reperfusion. Biochim Biophys Acta 1852: 262-270, 2015

10. Zhang L, Ding WY, Wang ZH, Tang MX, Wang F, Li Y, Zhong M, Zhang Y and Zhang W: Early administration of trimetazidine attenuates diabetic cardiomyopathy in rats by alleviating fibrosis, reducing apoptosis and enhancing autophagy. J Transl Med 14: 109-112, 2016

11. Guo R, Hu N, Kandadi MR and Ren J: Facilitated ethanol metabolism promotes cardiomyocyte contractile dysfunction through autophagy in murine hearts. Autophagy 8: 593-608, 2012.

12. van Rooij E, Sutherland LB, Liu N, Williams AH, McAnally J, Gerard RD, Richardson JA and Olson EN: A signature pattern of stress-responsive microRNAs that can evoke cardiac hypertrophy and heart failure. Proc Natl Acad Sci U S A 103: 18255-18260, 2006.

13. Jing L, Jin C, Lu Y, Huo P, Zhou L, Wang Y and Tian Y: Investigation of microRNA expression profiles associated with human alcoholic cardiomyopathy. Cardiology 130: 223-233, 2015.
14. Calvert JW, Coetzee WA and Lefer DJ: Novel insights into hydrogen sulfide - mediated cytoprotection. Antioxid Redox Signal 12: 1203-1217, 2010.

15. Zhou X, An G and Chen J: Hydrogen sulfide improves left ventricular function in smoking rats via regulation of apoptosis and autophagy. Apoptosis 19: 998-1005, 2014.

16. Zhang M, Shan H, Chang P, Wang T, Dong W, Chen X and Tao L: Hydrogen sulfide offers neuroprotection on traumatic brain injury in parallel with reduced apoptosis and autophagy in mice. PLoS One 9: e87241, 2014

17. Toldo S, Das A, Mezzaroma E, Chau VQ, Marchetti C, Durrant D, Samidurai A, Van Tassell BW, Yin C, Ockaili RA, et al: Induction of microRNA-21 with exogenous hydrogen sulfide attenuates myocardial ischemic and inflammatory injury in mice. Circ Cardiovasc Genet 7: 311-320, 2014.

18. Ge W and Ren J: mTOR-STAT3-notch signalling contributes to ALDH2-induced protection against cardiac contractile dysfunction and autophagy under alcoholism. J Cell Mol Med 16: 616-626, 2012.

19. Guo H, Sa Y, Huang J, Wang Z, Wang L, Xie M and Lv X: Urethral reconstruction with small intestinal submucosa seeded with oral keratinocytes and TIMP-1 siRNA transfected fibroblasts in a rabbit model. Urol Int 96: 223-230, 2016.

20. Johnson KM and Crocker SJ: TIMP-1 couples RhoK activation to IL-1 $\beta$-induced astrocyte responses. Neurosci Lett 609: 165-170, 2015.

21. Schoppet M and Maisch B: Alcohol and the heart. Herz 26: 345-352, 2001.

22. Laurent D and Edwards JG: Alcoholic cardiomyopathy: Multigenic changes underlie cardiovascular dysfunction. J Cardiol Clin Res 2: 1022, 2014.

23. Law BA, Levick SP and Carver WE: Alterations in cardiac structure and function in a murine model of chronic alcohol consumption. Microsc Microanal 18: 453-461, 2012.

24. Dancy M and Maxwell JD: Alcohol and dilated cardiomyopathy. Alcohol Alcohol 21: 185-198, 1986.

25. Wang Y, Wu Y, Chen J, Zhao S and Li H: Pirfenidone attenuates cardiac fibrosis in a mouse model of TAC-induced left ventricular remodeling by suppressing NLRP3 inflammasome formation. Cardiology 126: 1-11, 2013.

26. An Z, Yang G, He YQ, Dong N, Ge LL, Li SM and Zhang WQ: Atorvastatin reduces myocardial fibrosis in a rat model with post-myocardial infarction heart failure by increasing the matrix metalloproteinase-2/tissue matrix metalloproteinase inhibitor-2 ratio. Chin Med J (Engl) 126: 2149-2156, 2013.

27. Dixon IM, Ju H, Reid NL, Scammell-La Fleur T, Werner JP and Jasmin G: Cardiac collagen remodeling in the cardiomyopathic Syrian hamster and the effect of losartan. J Mol Cell Cardiol 29: 1837-1850, 1997.

28. Cowan KN, Jones PL and Rabinovitch M: Regression of hypertrophied rat pulmonary arteries in organ culture is associated with suppression of proteolytic activity, inhibition of tenascin-C, and smooth muscle cell apoptosis. Circ Res 84: 1223-1233, 1999.

29. Polyakova V, Loeffler I, Hein S, Miyagawa S, Piotrowska I, Dammer S, Risteli J, Schaper J and Kostin S: Fibrosis in endstage human heart failure: Severe changes in collagen metabolism and MMP/TIMP profiles. Int J Cardiol 151: 18-33, 2011.

30. Elrod JW, Calvert JW, Morrison J, Doeller JE, Kraus DW, Tao L, Jiao X, Scalia R, Kiss L, Szabo C, et al: Hydrogen sulfide attenuates myocardial ischemia-reperfusion injury by preservation of mitochondrial function. Proc Natl Acad Sci U S A 104: 15560-15565, 2007.

31. King AL and Lefer DJ: Cytoprotective actions of hydrogen sulfide in ischaemia-reperfusion injury. Exp Physiol 96: 840-846, 2011.

32. Qian H and Liu L: Protective effect of hydrogen sulfide on mice with experimental viral myocarditis and its mechanism. Xi Bao Yu Fen Zi Mian Yi Xue Za Zhi 30: 708-712, 2014 (In Chinese).

33. Zhou X, An G and Lu X: Hydrogen sulfide attenuates the development of diabetic cardiomyopathy. Clin Sci (Lond) 128: 325-335, 2015.

34. Zhou X, Lu X, Xu W and Chen J: Protective effects of hydrogen sulfide against chronic alcohol intake-induced left ventricular remodeling in rats. Cardiovasc Drugs Ther 27: 221-227, 2013.

35. Awata S, Nakayama K, Suzuki I and Kodama H: Effect of cysteine on the inactivation of cystathionine gamma-lyase by D,L-propargylglycine. Acta Med Okayama 43: 329-335, 1989.

36. Ashford TP and Porter KR: Cytoplasmic components in hepatic cell lysosomes. J Cell Biol 12: 198-202, 1962.

37. Zeglinski MR, Davies JJ, Ghavami S, Rattan SG, Halayko AJ and Dixon IM: Chronic expression of Ski induces apoptosis and represses autophagy in cardiac myofibroblasts. Biochim Biophys Acta 1863 (6 Pt A): 1261-1268, 2016. 
38. Matsui Y, Takagi H, Qu X, Abdellatif M, Sakoda H, Asano T, Levine B and Sadoshima J: Distinct roles of autophagy in the heart during ischemia and reperfusion: Roles of AMP-activated protein kinase and Beclin 1 in mediating autophagy. Circ Res 100: 914-922, 2007.

39. Baehrecke EH: Autophagy: Dual roles in life and death? Nat Rev Mol Cell Biol 6: 505-510, 2005.

40. Zhu H, Tannous P, Johnstone JL, Kong Y, Shelton JM, Richardson JA, Le V, Levine B, Rothermel BA and Hill JA: Cardiac autophagy is a maladaptive response to hemodynamic stress. J Clin Invest 117: 1782-1793, 2007.

41. Miyata S, Takemura G, Kawase Y, Li Y, Okada H, Maruyama R, Ushikoshi H, Esaki M, Kanamori H, Li L, et al: Autophagic cardiomyocyte death in cardiomyopathic hamsters and its prevention by granulocyte colony-stimulating factor. Am J Pathol 168: 386-397, 2006.

42. Xiao T, Luo J, Wu Z, Li F, Zeng O and Yang J: Effects of hydrogen sulfide on myocardial fibrosis and PI3K/AKT1-regulated autophagy in diabetic rats. Mol Med Rep 13: 1765-1773, 2016.

43. Shui M, Liu X, Zhu Y and Wang Y: Exogenous hydrogen sulfide attenuates cerebral ischemia-reperfusion injury by inhibiting autophagy in mice. Can J Physiol Pharmacol 22: 1-6, 2016.

44. Sun L, Jin H, Sun L, Chen S, Huang Y, Liu J, Li Z, Zhao M, Sun Y, Tang C, et al: Hydrogen sulfide alleviates myocardial collagen remodeling in association with inhibition of $\mathrm{TGF}-\beta / \mathrm{Smad}$ signaling pathway in spontaneously hypertensive rats. Mol Med 20: 503-515, 2015.

45. Lin L, Liu X, Xu J, Weng L, Ren J, Ge J and Zou Y: High-density lipoprotein inhibits mechanical stress-induced cardiomyocyte autophagy and cardiac hypertrophy through angiotensin II type 1 receptor-mediated PI3K/Akt pathway. J Cell Mol Med 19: 1929-1938, 2015

46. Wang ZG, Wang Y, Huang Y, Lu Q, Zheng L, Hu D, Feng WK, Liu YL, Ji KT, Zhang HY, et al: bFGF regulates autophagy and ubiquitinated protein accumulation induced by myocardial ischemia/reperfusion via the activation of the PI3K/Akt/mTOR pathway. Sci Rep 5: 9287, 2015.

47. Dany M, Rimmani HH, Matar SA and Hajj Hussein I: mTORC2-Akt signaling axis is implicated in myocardial compensation and fibrosis. J Biol Regul Homeost Agents 29: 745-753, 2015.

48. Usunier B, Benderitter M, Tamarat R and Chapel A: Management of fibrosis: The mesenchymal stromal cells breakthrough. Stem Cells Int 2014: 340257, 2014

49. Zhang Y, Wang H, Cui L, Zhang Y, Liu Y, Chu X, Liu Z, Zhang J and Chu L: Continuing treatment with Salvia miltiorrhiza injection attenuates myocardial fibrosis in chronic iron-overloaded mice. PLoS One 10: e124061, 2015.

50. Raaf L, Noll C, Cherifi MelH, Samuel JL, Delcayre C, Delabar JM, Benazzoug Y and Janel N: Myocardial fibrosis and TGFB expression in hyperhomocysteinemic rats. Mol Cell Biochem 347: 63-70, 2011.

51. Araki S, Izumiya Y, Rokutanda T, Ianni A, Hanatani S, Kimura Y, Onoue Y, Senokuchi T, Yoshizawa T, Yasuda O, et al: Sirt7 contributes to myocardial tissue repair by maintaining transforming growth factor- $\beta$ signaling pathway. Circulation 132: 1081-1093, 2015

52. Condorelli G, Latronico MV and Dorn GW II: MicroRNAs in heart disease: Putative novel therapeutic targets? Eur Heart J 31 $649-658,2010$
53. Wang M, Wang Y, Zang W, Wang H, Chu H, Li P, Li M, Zhang G and Zhao G: Downregulation of microRNA-182 inhibits cell growth and invasion by targeting programmed cell death 4 in human lung adenocarcinoma cells. Tumour Biol 35: 39-46, 2014.

54. Chen C, Jia KY, Zhang HL and Fu J: miR-195 enhances cardiomyocyte apoptosis induced by hypoxia/reoxygenation injury via downregulating c-myb. Eur Rev Med Pharmacol Sci 20: 3410-3416, 2016

55. Yu J, Wu SW and Wu WP: A tumor-suppressive microRNA, miRNA-485-5p, inhibits glioma cell proliferation and invasion by down-regulating TPD52L2. Am J Transl Res 9: 3336-3344, 2017.

56. Nouraee $\mathrm{N}$ and Mowla SJ: miRNA therapeutics in cardiovascular diseases: Promises and problems. Front Genet 6: 232, 2015.

57. Iacopo F, Lorenzo C, Calogero E, Matteo P, Riccardo PN, Veronica S, Valentina B, Riccardo L, Cristian S, Maria MC, et al: Review in translational cardiology: MicroRNAs and myocardial fibrosis in aortic valve stenosis, a deep insight on left ventricular remodeling. J Cardiovasc Echogr 26: 109-114, 2016.

58. Chen S, Puthanveetil P, Feng B, Matkovich SJ, Dorn GW II and Chakrabarti S: Cardiac miR-133a overexpression prevents early cardiac fibrosis in diabetes. J Cell Mol Med 18: 415-421, 2014.

59. Thum T, Gross C, Fiedler J, Fischer T, Kissler S, Bussen M, Galuppo P, Just S, Rottbauer W, Frantz S, et al: MicroRNA-21 contributes to myocardial disease by stimulating MAP kinase signalling in fibroblasts. Nature 456: 980-984, 2008.

60. Liang H, Zhang C, Ban T, Liu Y, Mei L, Piao X, Zhao D, Lu Y, Chu W and Yang B: A novel reciprocal loop between microRNA-21 and TGFßRIII is involved in cardiac fibrosis. Int J Biochem Cell Biol 44: 2152-2160, 2012.

61. Wang C, Wang S, Zhao P, Wang X, Wang J, Wang Y, Song L, Zou Y and Hui R: miR-221 promotes cardiac hypertrophy in vitro through the modulation of p27 expression. J Cell Biochem 113: 2040-2046, 2012.

62. Garofalo M, Di Leva G, Romano G, Nuovo G, Suh SS, Ngankeu A, Taccioli C, Pichiorri F, Alder H, Secchiero P, et al: miR-221\&222 regulate TRAIL resistance and enhance tumorigenicity through PTEN and TIMP3 downregulation. Cancer Cell 16: 498-509, 2009.

63. Cheng M, Wu G, Song Y, Wang L, Tu L, Zhang L and Zhang C: Celastrol-induced suppression of the miR-21/ERK signalling pathway attenuates cardiac fibrosis and dysfunction. Cell Physiol Biochem 38: 1928-1938, 2016.

64. Yu X, Li R, Shi W, Jiang T, Wang Y, Li C and Qu X: Silencing of microRNA-21 confers the sensitivity to tamoxifen and fulvestrant by enhancing autophagic cell death through inhibition of the PI3K-AKT-mTOR pathway in breast cancer cells. Biomed Pharmacother 77: 37-44, 2016.

65. Chen Q, Zhou Y, Richards AM and Wang P: Up-regulation of miRNA-221 inhibits hypoxia/reoxygenation-induced autophagy through the DDIT4/mTORC1 and Tp53inp1/p62 pathways. Biochem Biophys Res Commun 474: 168-174, 2016.

This work is licensed under a Creative Commons Attribution-NonCommercial-NoDerivatives 4.0 International (CC BY-NC-ND 4.0) License. 